\title{
428 PRELIMINARY CLINICAL RESULTS OF TORIPALIMAB MONOTHERAPY OR COMBINATION THERAPY FOR RECURRENT OR REFRACTORY GYNECOLOGIC CANCER
}

Peng Diao*, Qian Peng, Xingbo Luo, Qing Huang, Gaoshu Yan, Yan Tan. Sichuan cancer hospital, Chengdu, China

Background PD-1 inhibitor have demonstrated significant efficacy in the treatment of recurrent or refractory gynecologic cancer. However, the clinical data among Chinese patients is still limited. In this study, the efficacy and safety of toripali$\mathrm{mab}$ in the treatment of recurrent or refractory gynecologic cancer was evaluated.

Methods A retrospective analysis of patients with recurrent or refractory gynecologic cancer who were treated with toripalimab in Sichuan Cancer Hospital from September 2019 to December 2020 was performed. The treatment regimens included toripalimab monotherapy or toripalimab in combination with other therapies (eg, radiotherapy, chemotherapy, targeted therapy). Statistical analysis of the objective response rate(ORR), progression-free survival(PFS), and toxicity after the treatment was carried out.

Results In total 18 recurrent or refractory gynecologic cancer patients who received toripalimab were reviewed in this study, including 12 patients with cervical cancer, 3 patients with vulvar cancer, 2 patients with uterine tumor, and 1 patient with pelvic wall squamous cell carcinoma. The patients previously received $\leq 1$ line of treatment for advanced or recurrent cancer. The median follow-up period was 8 months [3.17-19.27 months]. The overall ORR was $33.3 \%$ (6/18), DCR was $77.8 \%$ (14/18), and median PFS was 8 months [95\% CI: 6.53-NA].For patients who received toripalimab as monotherapy, the ORR was $22.2 \%$ (2/9), DCR was $66.7 \%$ (6/9), and median PFS was 11.8 months (95\% CI: 6.53-NA). For patients treated with toripalimab combination therapy, the ORR was $44.4 \%$ (4/9), DCR was $88.9 \%$ (8/9), and median PFS was 8 months (95\% CI: 6.23-NA). Select treatment related AEs (TRAEs) of any grade were observed in $46.7 \%$ (7/ 18) patients. Grade 3 hepatotoxicity was recorded in 1 case. There was no treatment-related deaths.

Conclusions Toripalimab as monotherapy or Toripalimab combining with traditional anti-cancer therapy shows promising efficacy and acceptable toxicity in Chinese recurrent or refractory gynecologic cancer

http://dx.doi.org/10.1136/jitc-2021-SITC2021.428 